\title{
Grouping and detection of group B streptococci by immunofluorescence
}

\author{
D CASTLE, JANE DEELEY, CSF EASMON
}

From the Department of Medical Microbiology, Wright-Fleming Institute, St Mary's Hospital Medical School, London W2 1PG

SUMMARY Forty-nine clinical isolates of group B streptococci (GBS) were correctly grouped from broth culture by the Fluoro-Kit immunofluorescence test. A further 82 beta-haemolytic streptococci of groups A, C, D, F, and G were tested and gave no cross-reactions. The test was simple to perform and gave clear results. The Fluoro-Kit reagents, however, failed to detect GBS in 21 $(51 \%)$ of 41 smears of rectal or vaginal swabs from pregnant women from which GBS were subsequently grown. Thirty-two $(20 \%)$ of 159 culture-negative swabs gave positive immunofluorescent reactions.

Group B streptococci (GBS) are important neonatal pathogens. Effective specific therapy depends on rapid diagnosis, as infection in the first few days of life which carries the highest mortality, usually follows heavy superficial GBS colonisation. ${ }^{1}$ The organisms are acquired at birth from the mother's genital tract. ${ }^{1}$ The rapid detection of heavy maternal and infant GBS colonisation from superficial swabs and gastric aspirate would allow preventive measures to be taken where necessary. Culture is not sufficiently rapid. Non-specific microscopy such as the Gram stain may prove useful, but is often not sensitive enough. Immunofluorescence microscopy could be both rapid and sensitive if suitable reagents were available. In this paper we have examined the efficiency of an immunofluorescence kit (FluoroKit-Clinical Sciences Inc) for the identification of GBS in pure culture and for the detection of GBS from rectal and vaginal swabs from pregnant women.

\section{Material and methods}

Streptococcal strains of groups A, B, C, D, F, and G were isolated from clinical material and grouped using Streptex (Wellcome Reagents). Smears were made from 200 rectal or vaginal swabs taken from pregnant women as part of a larger study on GBS carriage during pregnancy. Smears were fixed in

Accepted for publication 1 December 1982
$70 \%$ methanol and stored for immunofluorescence testing. The same swabs were used to inoculate Columbia blood agar and selective Islam's agar plates, ${ }^{2}$ the latter containing nalidixic acid $15 \mathrm{mg} / \mathrm{l}$ and gentamicin sulphate $4 \mathrm{mg} / 1$. The plates were incubated anaerobically overnight at $37^{\circ} \mathrm{C}$. Swabs were finally placed in Todd-Hewitt broth (Oxoid) supplemented with the same antibiotics and after overnight incubation, subcultured onto blood and non-selective Islam's agar.

Pure cultures of the various streptococcal strains to be grouped were grown up in Todd-Hewitt broth. Fluoro-Kit slides were removed from storage at $-20^{\circ} \mathrm{C}$ and a loopful of culture placed on each of the seven wells and the inoculated slides air dried at room temperature. Each slide had a further three wells containing a positive group B control and negative group A and Staphylococcus aureus controls. The slides were fixed for one minute with the fixative provided and again air-dried. Fluorescein conjugated group B reagent $(0.02 \mathrm{ml})$ was applied to each well and the slides incubated in a moist dark chamber at room temperature for $30 \mathrm{~min}$. The slides were washed twice for five minutes in phosphatebuffered saline, rinsed in distilled water, dried, mounted and examined immediately with a Leitz Dialux fluorescence microscope. The rectal and vaginal smears were treated in the same way except that they were prefixed on ordinary glass slides. Using the instructions given with the kit, negative reactions were taken as those which gave either no fluorescence of + or ++ reactions. Positive reactions were taken as those giving +++ or ++++ fluorescence. 
Table 1 Immunofluorescence reactions of streptococci

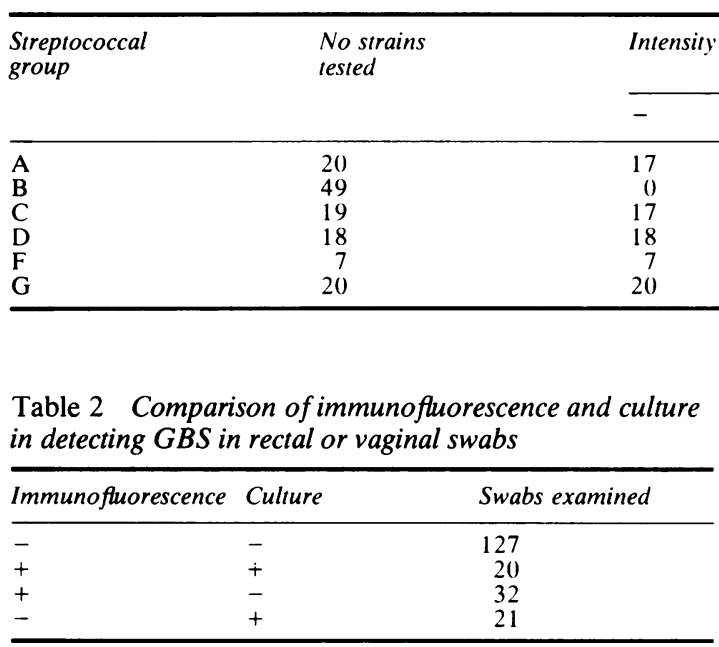

\section{Results}

One hundred and thirty-one streptococcal isolates were grouped by Fluoro-Kit; the results are shown in Table 1. All 49 group B isolates gave ++++ reactions. Most of the other streptococci did not fluoresce while a few gave + reactions. The slides were simple to read.

Table 2 shows the results obtained with rectal and vaginal smears. In $147(73.5 \%)$ of 200 smears, there was agreement between culture and immunofluorescence. Of the $53(26.5 \%)$ where there were disagreements, 32 were culture-negative with positive microscopy and 21 culture-positive with negative microscopy. Bacteria appeared as coccobacilli, often in short chains. There was an appreciable amount of background fluorescence, but this caused no problems in interpretation. Ten samples were tested a second time to test reproducibility. Nine of the ten gave the same result (6 positive, 3 negative). The tenth gave a positive reaction on the first occasion and a negative reaction on the second.

\section{Discussion}

As a grouping technique for group B streptococci from primary culture, the Fluoro-Kit worked extremely well. The reactions were clear cut and simple to read with no cross-reactions from the other major streptococcal groups.

As a rapid diagnostic test for the presence of GBS in smears from vaginal swabs, Fluoro-Kit was less satisfactory. Of the 41 swabs that were positive for GBS by culture, $21(51 \%)$ were negative by immunofluorescence. Conversely $32(20 \%)$ of 159

culture negative swabs were positive by승 immunofluorescence. The 21 culture-positive fluorescence negative samples are the more worry-o ing as with these proven GBS colonisation would have been missed. Eleven of the 21 swabs were $\overrightarrow{\mathrm{G}}$ GBS-positive on enrichment culture only, indicatingo scanty GBS carriage. It is possible that the small numbers of bacteria present were either missed dur- $\rightarrow$ ing microscope examination, not transferred to the slide during preparation of the smear, or not fixed $\overrightarrow{ }$ properly. This would not, however, explain the remaining 10 culture-positive fluorescence negative ${ }^{\omega}$ smears where swabs grew on primary culture.

It is more difficult to comment on the 32 cultures negative fluorescent positive samples. IgG reagentsō can combine non-specificity with protein A containing Staph aureus and indeed the Fluoro-Kit slides@ contain a staphylococcal control. Bacterial mor- $\overrightarrow{\vec{B}}$ phology in the positive smears did not suggest 3 staphylococci. Even with enrichment techniques? some cultures may have failed to grow small num-: bers of GBS. Six of the 32 samples were from women who had positive cultures at other times during pregnancy. The other 26 were consistently culture-negative. Immunofluorescence could also have detected bacteria which had been killed either 3 by antimicrobial therapy or by local chlorhexidine used during vaginal examination. Only two of the 32 글 women were or had recently been on antimicrobialo therapy. We did attempt to take samples before any chlorhexidine was used locally, but we cannot be $N$ sure that this was done in all cases.

We can perhaps be criticised for using ordinary slides and methanol fixation. However, the specially $y_{0}^{\omega}$ prepared Fluoro-Kit slides have seven test wells and it would seem neither practical nor economic to use only one or two of these. Used directly on smears the Fluoro-Kit immunofluorescence reagents do not appear sufficiently sensitive for the early detection of GBS colonisation. A compromise might be to cul- $\frac{\mathrm{\Phi}}{\mathrm{\phi}}$ ture the swabs in broth for 3-4 hours and to use the broth culture as material for immunofluorescence testing. This would, of course, delay the diagnosis and if this approach was used other detection 
methods for GBS such as latex or coagglutination might prove simpler and more economical.

We thank the Medical Research Council for financial support.

\section{References}

' Ancona RJ, Ferrieri P, Williams PP. Maternal factors that enhance the acquisition of group $B$ streptococci by newborn infants. J Med Microbiol 1980;13:273-80.

${ }^{2}$ Islam AKMS. Rapid recognition of group B streptococci. Lancet 1977;i:256-7.

Requests for reprints to: Dr CSF Easmon, Department of Medical Microbiology, Wright-Fleming Institute, St Mary's Hospital Medical School, London W2 1PG, England. 